Journal Club

Editor's Note: These short reviews of a recent paper in the Journal, written exclusively by graduate students or postdoctoral fellows, are intended to mimic the journal clubs that exist in your own departments or institutions. For more information on the format and purpose of the Journal Club, please see http://www.jneurosci.org/misc/ifa_features.shtml.

\title{
It Is Time to Move: Role of Lateral Diffusion in AMPA Receptor Trafficking
}

\author{
Guang Yang, Alan Huang, Shanshan Zhu, and Wei Xiong \\ Graduate Program of Neuroscience and the Brain Research Centre, University of British Columbia, Vancouver, British Columbia, Canada V6T 2B5 \\ Review of Ashby et al. (http://www.jneurosci.org/cgi/content/full/26/26/7046)
}

AMPA receptors (AMPARs) contribute to synaptic plasticity by exocytosis and endocytosis of receptors between membrane and intracellular pools (Collingridge et al., 2004). Single-molecule fluorescence microscopy also demonstrates that surface AMPARs may diffuse laterally to extrasynaptic sites where they cycle with intracellular pools (Borgdorff and Choquet, 2002). A recent paper in the Journal of Neuroscience (Ashby et al., 2006) investigated the role of lateral diffusion in AMPAR constitutive trafficking, and demonstrate that spine morphology affects lateral movement.

To selectively visualize surfaceexpressed AMPARs, the investigators tagged glutamate receptor subtype 2 (GluR2) subunits with the $\mathrm{pH}$-sensitive green fluorescent protein [superecliptic pHluorin (SEP)]. SEP loses fluorescence in an acidic environment but is bright at neutral $\mathrm{pH}$. Because vesicular internal organelles containing AMPARs are acidified, bright fluorescence, therefore, should be detected only from surface SEPGluR2s exposed to the extracellular area [Figure 1; Ashby et al. (2006), their Fig. 1A-C (http://www.jneurosci.org/cgi/ content/full/26/26/7046/F1)]. Interestingly, acidic wash completely quenched

Received July 17, 2006; revised Aug. 1, 2006; accepted Aug. 1, 2006. Correspondence should be addressed to Guang Yang, Graduate Program of Neuroscience and the Brain Research Centre, University of British Columbia, Vancouver, British Columbia, Canada V6T 2B5. E-mail: photonyg@interchange.ubc.ca.

DOI:10.1523/JNEUROSCI.3018-06.2006

Copyright $\odot$ 2006 Society for Neuroscience $\quad$ 0270-6474/06/269082-02\$15.00/0 fluorescence from the spine head, whereas alkalinization that increased internal $\mathrm{pH}$ had no effect on spine fluorescence [Ashby et al. (2006), their Fig. $1 E$ (http://www. jneurosci.org/cgi/content/full/26/26/7046/ F1)], suggesting that most of the tagged AMPARs in the spine head were on the surface. Using fluorescence recovery after photobleaching (FRAP), the authors noticed that the recovery of fluorescence in a single spine soon reached a plateau, implying constitutive cycling of AMPARs on the spine surface [Ashby et al. (2006), their Fig. 1A,B (http://www.jneurosci.org/cgi/content/full/ 26/26/7046/F1)]. To exclude the possibility that on-off cycling between the membrane and intracellular pools also contributed to the FRAP, the authors performed antibody cross-linking. This procedure blocked lateral movement of membrane AMPARs and correspondingly the FRAP [Ashby et al. (2006), their Fig. 2C (http://www. jneurosci.org/cgi/content/full/26/26/7046/ F2)]. In contrast, exocytosis of AMPARs from intracellular pools was intact in the presence of antibody in the matrix [Ashby et al. (2006), their Fig. 2E,F (http://www. jneurosci.org/cgi/content/full/26/26/7046/ F2)]. Indeed, exocytosis of AMPARs was unable to rescue the blockade of FRAP by antibody cross-linking [Ashby et al. (2006), their Fig. 2 (http://www.jneurosci.org/cgi/ content/full/26/26/7046/F2)], indicating that constitutive AMPAR exchange was driven by lateral diffusion.

A comparison of FRAP in spiny and nonspiny regions showed that AMPAR movement was slower in spines [Ashby et al. (2006), their Table 1 (http://www. jneurosci.org/cgi/content/full/26/26/7046/ T1)]. The slow diffusion rate of AMPARs on the spiny membrane could be attributable to special spine structures. To test this hypothesis, the authors focused on the narrow neck region of spines and monitored fluorescence loss in photobleaching from adjacent regions of the spine head. If the neck region acts as a barrier to AMPAR diffusion, one would expect a different time course of fluorescence loss between neck and head. Indeed, continually bleaching the spine head caused rapid fluorescence loss in regions adjacent to the spine head. In contrast, spine necks showed a relatively slow loss of fluorescence [Ashby et al. (2006), their Fig. 4A (http://www.jneurosci.org/cgi/content/ full/26/26/7046/F4)], suggesting a role for the spine neck in restricting lateral diffusion of AMPARs.

If narrow spine necks actually act as a barrier, stubby spines without necks should display faster fluorescence recovery rates after bleaching of their spine heads. In fact, the neck of mushroom spines caused a 2.4 times slower diffusion rate [Ashby et al. (2006), their Fig. 4B,C (http://www.jneurosci.org/cgi/content/ full/26/26/7046/F4)]. These data clearly demonstrate that the spine morphology affects AMPAR lateral diffusion on synaptic membranes. The neck structure in spines seemed to restrict the movement of other membrane-associated molecules as well, suggesting a general function of the spine neck in compartmentalizing mem- 

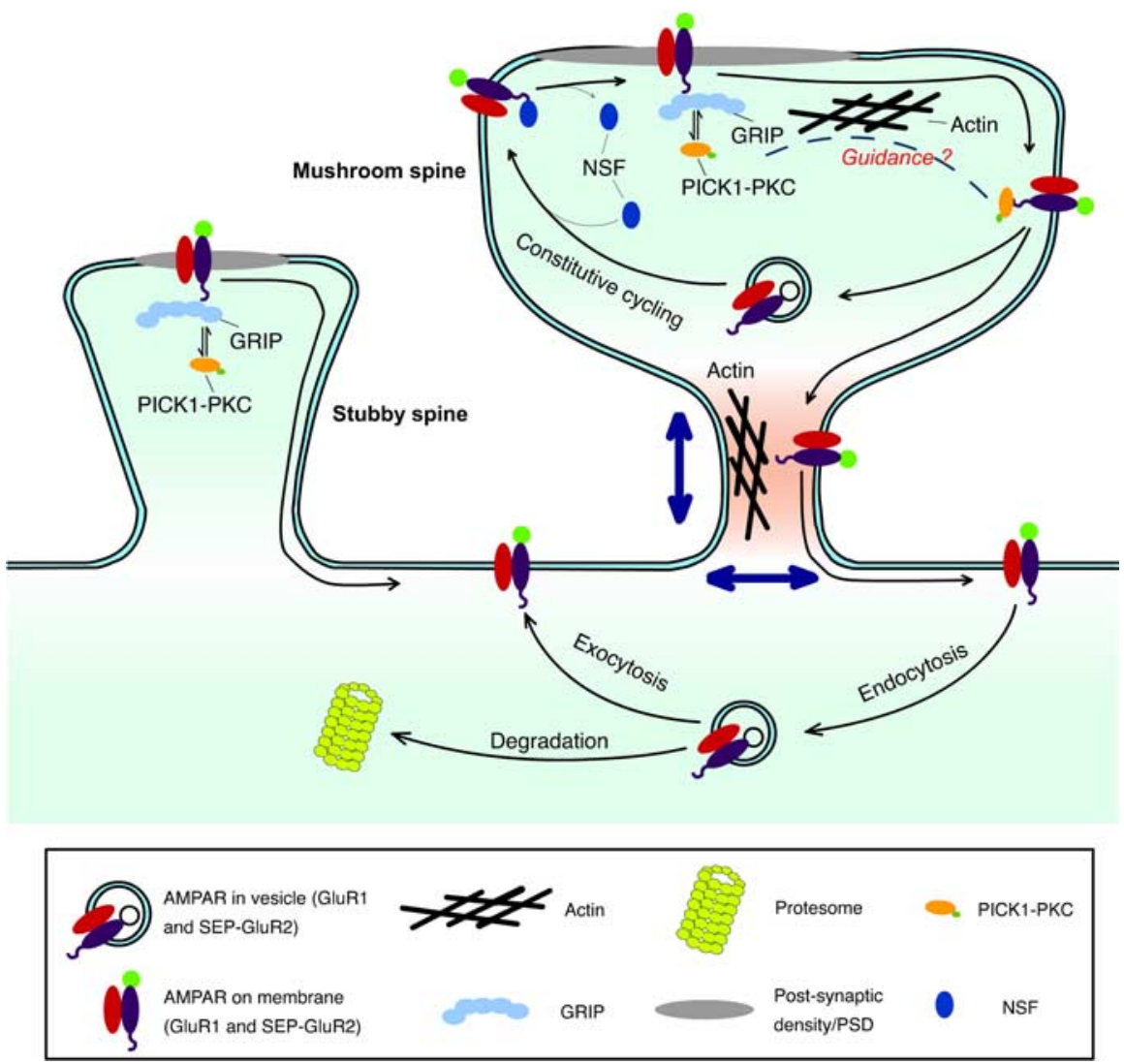

Figure 1. The model depicts AMPARs trafficking in dendritic spines. GluR2-containing AMPARs constitutively cycle within spines, regulated by proteins that directly bind to AMPARs. For example, a PDZ domain-containing protein GRIP stabilizes AMPARs on the surface and can be replaced by a PICK1-PKC complex that facilitates AMPAR internalization. Before endocytosis, AMPARs move laterally from the synaptic site to extrasynaptic regions, and then internalize. Constitutive cycling AMPARs may insert by exocytosis at extrasynaptic areas and then diffuse laterally to the synapse. NSF may play an important role in this process by freeing GluR2 from extrasynaptic membrane anchors (Gardner et al., 2005). How AMPARs are guided during diffusion is not clear. Diffusing AMPARs could also move out of the spines through the narrow spine neck. The diffusion rate of AMPARs is much slower at necks (shown by the red color) compared with the spine head (light blue). Spines without necks also show a relatively rapid diffusion rate. Therefore, the actin skeleton could regulate AMPAR lateral diffusion by altering the spine neck (shown as the double-headed arrow).

brane molecules [Ashby et al. (2006), their Fig. 5 (http://www.jneurosci.org/cgi/ content/full/26/26/7046/F5)].

Although exocytosis and endocytosis have been considered the main factors regulating constitutive and activitydependent AMPAR trafficking at dendritic spines, an emerging model suggests that lateral diffusion into and from synaptic sites is a necessary step during AMPAR trafficking (Triller and Choquet, 2005). Ashby et al. (2006) demonstrate that lateral diffusion of AMPARs, rather than directed exocytosis, can drive AMPAR con- glutamate receptor interacting protein (GRIP) and protein interacting with $C$ kinase 1 (PICK1) interact with PDZ-domains on AMPAR and are important for AMPAR trafficking. Release of AMPARs from another interacting protein, $\mathrm{N}$-ethylmaleimide-sensitive factor (NSF), promotes trafficking to the postsynaptic density from extrasynaptic sites (Collingridge et al., 2004). It is not clear whether these proteins also guide to help AMPARs to their target sites during lateral diffusion (Fig. 1), or if protein interactions serve as tracks for lateral movement. AMPARs could also move randomly after release from membrane anchors (Gardner et al., 2005). AMPARs may also be regulated by links to the actin cytoskeleton (Zhou et al., 2001). This is interesting when considering the contribution of actin to dendritic spine morphology (Matus, 2000). The dynamic change of neck structure by actin is a potential factor regulating AMPAR lateral movement during plasticity (Fig. 1). Examination of the involvement of actin in lateral diffusion regulation may provide additional insights into the relationship between spine morphology and AMPAR trafficking.

\section{References}

Ashby MC, Maier SR, Nishimune A, Henley JM (2006) Lateral diffusion drives constitutive exchange of AMPA receptors at dendritic spines and is regulated by spine morphology. J Neurosci 26:7046-7055.

Borgdorff AJ, Choquet D (2002) Regulation of AMPA receptor lateral movements. Nature 417:649-653.

Collingridge GL, Isaac JT, Wang YT (2004) Receptor trafficking and synaptic plasticity. Nat Rev Neurosci 5:952-962.

Gardner SM, Takamiya K, Xia J, Suh JG, Johnson R, Yu S, Huganir RL (2005) Calciumpermeable AMPA receptor plasticity is mediated by subunit-specific interactions with PICK1 and NSF. Neuron 45:903-915.

Matus A (2000) Actin-based plasticity in dendritic spines. Science 290:754-758.

Triller A, Choquet D (2005) Surface trafficking of receptors between synaptic and extrasynaptic membranes: and yet they do move! Trends Neurosci 28:133-139.

Zhou Q, Xiao M, Nicoll RA (2001) Contribution of cytoskeleton to the internalization of AMPA receptors. Proc Natl Acad Sci USA 98: 1261-1266. 